\title{
Medical Hypotheses
}

Medical Hypotheses (1988) 27, 167-179

(C) Longman Group UK Led 1988

\section{Reflections on the Pathogenesis of Optic (Retrobulbar) Neuritis in Multiple Sclerosis}

\author{
R.U. SCHWYZER* and HUGO HENZI \\ *Incella, CH 6614 Brissago, Switzerland. Freihans 4, CH 8184, Bachenbülach, Switzerland.
}

\begin{abstract}
The conditions which lead to a plaque of demyelination in the retrobulbar optic nerve are discussed. Growth of the plaque occurs along venules as small fingerlike sleeves which develop outwards from the contour of the plaque. This occurs slowly and at intervals; the very gradually expanding lesion remain for a long time clinically silent.

It is here postulated that the change to clinical disease is induced if cells digesting myelin debris settle in a more distal part of the perivascular space of a vein, than during the subclinical phase. In sufficient numbers these cells will impede the movement of molecules from extra-cellular fluid surrounding nodes of Ranvier into cerebrospinal fluid. A restriction in this vital drainage pathway results in oedema causing distrubed signal transmission in neurons passing through the veins drainage territory. Depending on intensity this can induce the characteristics symptom of blurred vision.

These concepts have been used to speculate on sequential changes in neurons and to relate them to various phases of the disease. This seems to be justified as the pattern evolved corresponds well with the clinical symptomatology.
\end{abstract}

\section{Introduction}

The argument here presented is based on the concept, that certain low molecular weight subtances, albeit present in health, may be the root cause of multiple sclerosis (MS) $(11,13)$.

It has been postulated that if methanol occurs at hypernormal quantity in systemic circulation it is able to induce a toxic allergic process in the human central nervous system (CNS) (12). If the abnormal stimulus occurs in repetitive episodes over a period of many months of years, the process may lead to the formation of plaques of demyelination.

The development of a plaque in the optic nerve is a frequent occurrence. Since the advent of testing patients with visual evoked potentials and other non-invasive methods it seems that most or all MS patients have some abnormality in the visual system $(6,7,9,23)$. Pathologist's opinion is $(18, \mathrm{p} 149)$, that MS cases typically show plaques in optic nerves, chiasma or optic tracts. In the vast majority of cases the plaques are situated in the retrobulbar optic nerve. 
Presumably this vulnerability of the optic nerve $(2,35)$ will become explicable once the pathologic process is thoroughly understood; at the moment we must call it: 'Unknown affinity between agent and tissue which leads to pathocline selection of damage' $(12, \mathrm{p} 12)$.

The methodological aspect of the methanol hypothesis had been discussed elsewhere (12, p 34). A survey of research findings has yielded a detailed model of plaque growth (29) which is here referred to as the MeHyp-model.

\section{Background}

Lumsden (18, p 597) made a remark which we have used as a guide-line: 'it is necessary to distinguish between ... the primary sensitization events that initiate the multiple sclerosis process as distinct from the multiple sclerosis plaque'.

Sensitization has been demonstrated in an involuntary experiment on R.G. R.G. periodically carried out certain experiments, which exposed him for periods, lasting for several hours, to the vapour of technical formaldehyde, and thus of methanol (technical formaldehyde contains $16 \%$ of methanol for stabilization purposes). The repeated exposure led to short term symptoms which appeared to be caused by the poisoning, however after $1 \frac{1}{2}$ years the pathologic picture of MS developed. One of the early symptoms of MS was blurred vision; the medical and ophthalmological reports described the usual symptoms and signs of optic neuritis (ON). The autopsy report gave the picture of an MS that had run its course (13).

The disease history of R.G. started with extended periods of 'methanol in blood' due to the intoxication at work. Under the term 'methanol in blood' we understand a level of methanol in the systemic circulation considerably exceeding the homeostatic value. Abnormal concentrations of methanol can be detected in the circulation following: 1) inhalation, 2) skin absorption, 3 ) ingestion (which would include endogenous formation of methanol derived from good ingested).

That some methanol is continuously excreted by the body has been known for many decades (12, p 17) but information about methanol homcostasis scems to be scanty. In a group of 17 men and 14 women recently investigated the mean level of methanol in urine was $0,73 \mathrm{mg} /$ liter, with extreme values of $0,32 \mathrm{mg} / 1$ and $2,61 \mathrm{mg} / \mathrm{l}$. When individuals were exposed to air containing
$300 \mathrm{mg} / \mathrm{m}^{3}$ methanol vapours for 8 hours, the content in urine rose gradually to between 8,5 and $10,5 \mathrm{mg} / 1$ by the end of this period. As methanol distributes in the body pro rata with the water content of tissues, blood levels during exposure must at times have been equally elevated. The exposure was maintained for a working shift and then discontinued. 8 hours later, i.e. 16 hours after first exposure, the methanol level was again within the normal range (30). Similar tests have been conducted by another group (4) with similar results. It has been suggested that urine measurements should be used to monitor workers and that values above $10 \mathrm{mg} / \mathrm{l}$ should lead to scrutiny of safety measures in use.

From these experiments and the recorded details of the case R.G. (13) we conclude that his methanol homeostasis was similarly temporarily disturbed. In the laboratory he inhaled methanol which entered through the lungs and reached the capillary blood.

The pathogenic events which are postulated to have induced MS in R.G. are: i) Methanol present in systemic circulation will diffuse into the extracellular fluid (ECF) as shown in Figure 1. Figure 1 introduces the term 'molecules characterizing pathology' (MCP). These will at the beginning of the disease process be mainly the diffusing methanol molecules. As will be seen when discussing Figure 2, the term has its advantages.

ii) In biological membranes and in the cytoplasm of cells the simultaneous presence of methanol (and its metabolite formaldehyde) and fructose (and its metabolite glyceraldehyde) can lead to complexities such that formaldehyde in a nascent state can be delayed in its further degradation (competitive inhibition) $(12$, p 20,29$)$.

iii) If this occurs the same process as in formol fixation must be expected. Formaldehyde will immediatly react with nearby proteins, i.e. attach itself to the $\mathrm{NH}_{2}-$ groups of side chains of amino acids (12, p 44).

iv) Wherever such changed proteins are not swiftly replaced, they will be recognized by the immune system as 'non-self' molecules and and allergic reaction will ensue (29).

Myelin, which has formed as an extension of the oligodendrocyte membrane, has a slow molecular replacement rate and there the 'nonself' molecules may exist for a sufficient length of time to stimulate an immune response. 


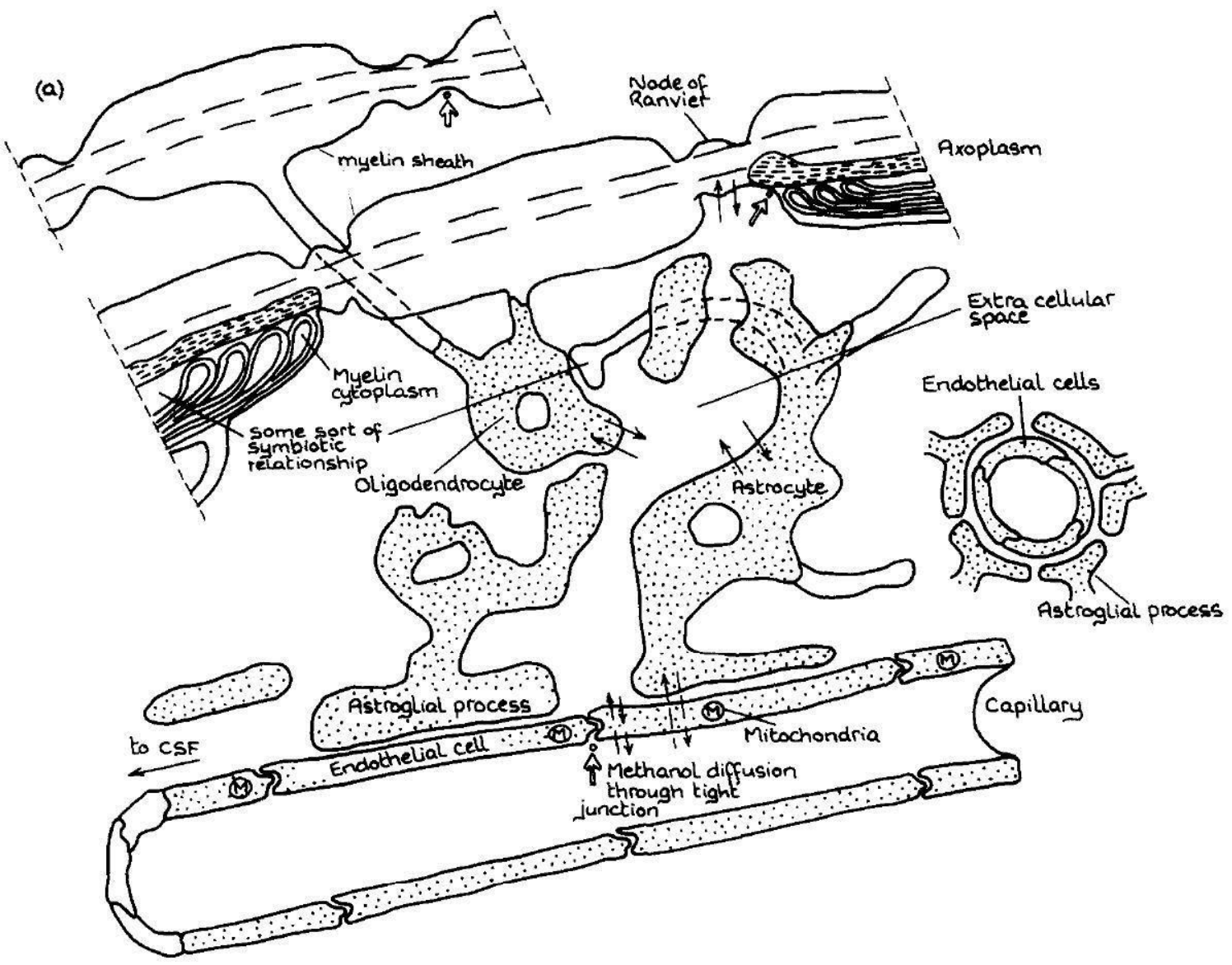

(b)

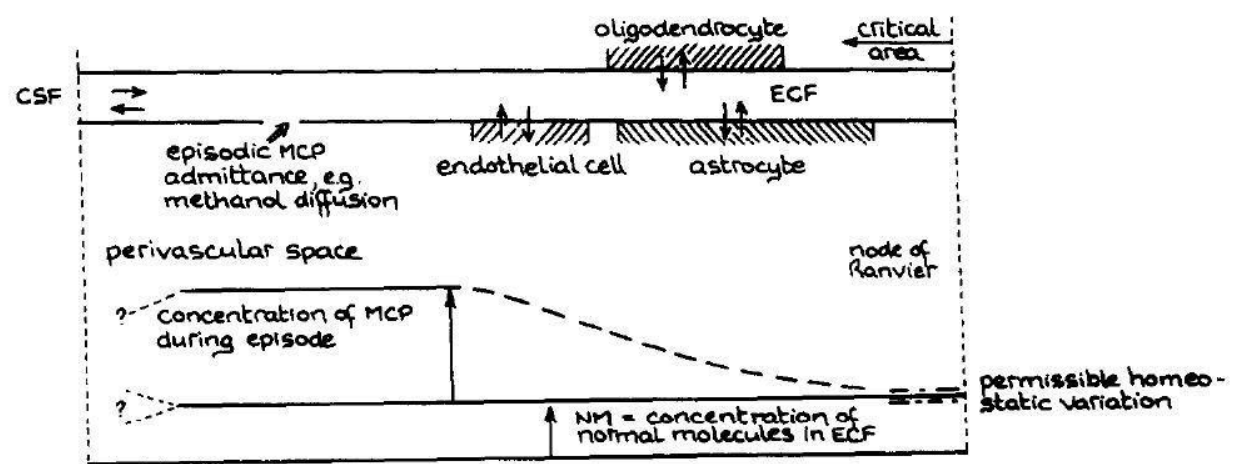

Fig 1 Cleft forming a direct pathway from capillary to node of Ranvier

(a) is a map showing spatial relationship, dimensions not in proportion, adapted from Kuffler and Nicholls (1976) (16) The cleft approx. $20 \mathrm{~nm}$ wide forms an aqueous extracellular pathway open to diffusion. Extra cellular fluid (ECF) influenced by movement as indicated hy black arrows and diffusion to and from cerebrospinal fluid (CSF). Open arrow indicated additional movement in pathology (see text).

(b) shows the principle of the channel formed by the cleft and its continuation into the perivascular space. Astrocytes can influence ECF by ingestion and/or ionic pumping (outward flow of fluids) that will flush undesirable molecules towards drainage.

(c) shows the distribution of concentration of 'molecules characterizing pathology' (MCP) over the length of the channel as per (b). Experimental evidence (19) gives some support of the relative length of ordinate shown at node of Ranvier and at the perivascular space.

The heavy broken line represents the influence of molecular pumping on chemical microclimate as necessary to maintain MCP at the node of Ranvier with permissible homeostatic limits 


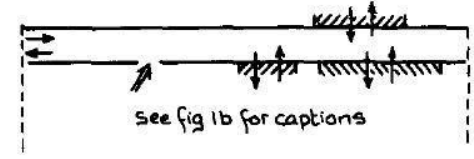

Stages

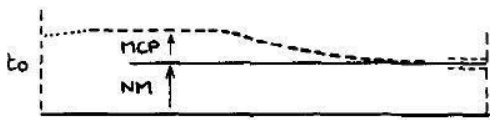

(A) increasing level of oxidative enzymatic activit (O) norm
of do.

Activity of cells astrocytes (A) oligodendrocytes (O) indicating level of molecules character ising pathology (MCP) at node of Rantier zones intersected at right angles to contour stages (sex fig's
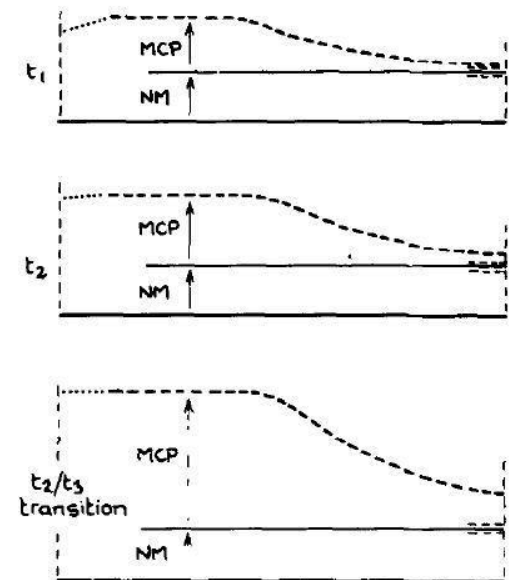

$t_{3} / t_{4}$ the cleft is changing transition
(A) as above (O) MCP penetration of membranes, attenuation exceeding replacement. start of degeneration

(A) hyperactive, intense molecular pumping and (O) as above.

(A) as above, but atso enlarged astrocytes containing reaction products and IgG

(O) as above. symbolic repre- data on myelin sentation of and axon data on axoplasm sodlum pump (see key)

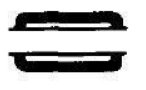

no abnormality

$\underset{++}{\text { normal }}$

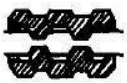

swelling, certain proteins change in conformation

compression causes movement to slow down

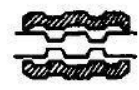

edema and immune reaction cause thinning of sheath

movement slowing down

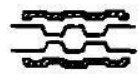

myalinalysis

stasis
0 ,

A) activity continues at high level

(O) degeneration proceeding, astrocyte processes

filling space formerly oc-

cupied by oligodendrocytes

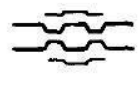

myelinolysis progressing

Fig 2 Portrayal of changes caused by MCP (molecules characterizing pathology) during 'methanol in blood episode' The diagram on top left of centre is a repeat of fig. $1 \mathrm{~b}$, consult explanations under $1 \mathrm{~b}$ and $1 \mathrm{c}$. On the graphs below the diagram the surmised distribution of MCP over the length of the cleft is shown. No scale is given for the ordinate. Absolute values are not available, relative values have been chosen in line with blood methanol values in an experiment $(19,29) ; t_{0}$ to $t_{4}$ denote stages through which a cleft passes as the plaque grows.

Note:

(i) NM denotes normal molecules at node of Ranvier under homeostatic conditions.

(ii) the chmical microclimate in the clef during $t_{1}$ and $t_{2}$ is considered to be responsible for the minute change during the first step of demyelination (swelling of myelin, compression of axon, change in conformation of protein in myelin, start of immune reaction, i.e. thinning of myelin sheath).

(iii) the chemical microclimate in the cleft at $t_{2}$ to $t_{3}$ transition in considered to be responsible for the beginning of the myelinolysis in the 2 nd step or demyelination.

(iv) near the centre of the plaque scar-tissue has formed in the area of the clefts.

(v) the MCP value at nodes of Ranvier of individual neurons situated along a line at right angle to the contour of the plaque is shown in the diagram of top right of centre. 
(vi) the stages $t_{0}$ etc. and the information to the right of the diagrams refer to the tissue condition as existing at the height of the 'methanol in blood episode'. When the episode has passed MCP does not entirely disappear (oedema persists) and change due to the immune reaction continues (see fig. 4d).

(vii) in fig. 1 astrocytes are shown to about directly on the capillaries as is normal in CNS. In the optic nerve capillaries and venules are situated within the pial septa and blood vessels are surrounded by collagen tissue, which in turn is bounded by the astrocytes. For ion exchange during signaling, ECF and a pathway for the return of tissue fluids must exist. Movement of molcculcs etc. is assumed to occur, same as in white matter, through perivascular space (2). In the diagram on top left of centre the diffusion pathway into CSF is indicated by the black arrow at left.

The antigen forming in myelin during the repetitive poisoning period may have sensitized R.G. for life to the toxic-allergic disease that followed.

R.G.'s case of MS was accepted on circumstantial evidence as the sequel of chronic methanol poisoning by the court who had to decide on his compensation claim. Nowadays with the progress made in mebrane research and other fields the court could in addition be supplied with a coherent biological explanation.

The further steps in the somewhat complex toxic-allergic disease process that may follow chronic methanol poisoning can be collated from MS research findings. To apply these findings we have first to consider whether the same condition, namely repetitive episodes of methanol in the lumen of the capillaries, could occur in MS patients.

In the MeHyp-model (12, p 15) the well known fact has been documented that polygalaturonic acid esterified with methanol is a constituent of what is referred to as pectin. Pectin is a common dietary ingredient, naturally in fruits and vegetables and frequently utilized by housewifes and by industry in food processing. The methylgroups of pectins are released in the intestincs and methanol enters the liver via the portal vein. If a high fructose ingestion occurs simultaneously with pectin the capacity of the liver to process methanol and fructose may be temporarily overtaxed. Thus a metabolic complication results, in which these low molecular weight substances enter the systemic circulation in above normal quantity; that is: a 'methanol in blood' episode can be brought about without the individual inhaling or drinking methanol as such.

Figure 2 summarizes the changes which occur at certain sites in the CNS during the toxicallergic disease which can follows as a consequence of the above. The time sequence called stages $t_{10}$ to $t_{4}$ and the diagrams are an attempt to show the dynamics of the process. The information is adapted from Figure 4 in (29); the concepts have developed from correlating facts and ideas about MS plaques. Some insights gleaned which are relevant to this discussion are:
A. Apart from methanol diffusion the increasing and changing cellular activity (Fig. 1) during the immune reaction in the cleft influences the composition of ECF and increases its volume. This ECF is then flushed out through the perivascular space which acts as a lymphatic channel (28). Simultaneously electrolyte concentration near nodes of Ranvier may be affected and even small changes can have a marked influence on conduction velocity (9).

B. Due to the ongoing immune reaction macrophages, lympocytes etc., accumulate in large numbers in the peri-vascular space (Fig. 5) (26). Further it is known that the macrophages may remain there for up to a year (1) digesting myelin debris. During this time. due to further episodes, new waves of mobile cells may appear. The deduction, that they may exert a bottleneck effect in the drain leading to CSF seems to be well based (29). Slower drainage will have the effect shown in the diagrams in Figure 2, i.e. increased MCP at node of Ranvier. Apart from methanol, formate, various organic acids and lysosomal enzymes are present and are capable of interfering with ATP production in the mitochondria of the axons (22).

C. Electron microscopy have shown immune competent cells associated with myelin breakdown and proliferation of astrocytes in biopsy specimens from MS patients (3). These minute changes in macroscopically normal white matter outside the plaque contour are considered to be the hallmark of morbid and premorbid MS (29). The immune system follows its normal defensive role by treating a new substance (new because protein or glycoprotein in myelin has been changed, see iv above) as an allergen. In later 'methanol in blood' episodes further minute changes occur and the intense activity of the immunological defence mechanism (26, 34) brings about the demyelination pattern that characterizes the lesion, i.e. plaque formation. 


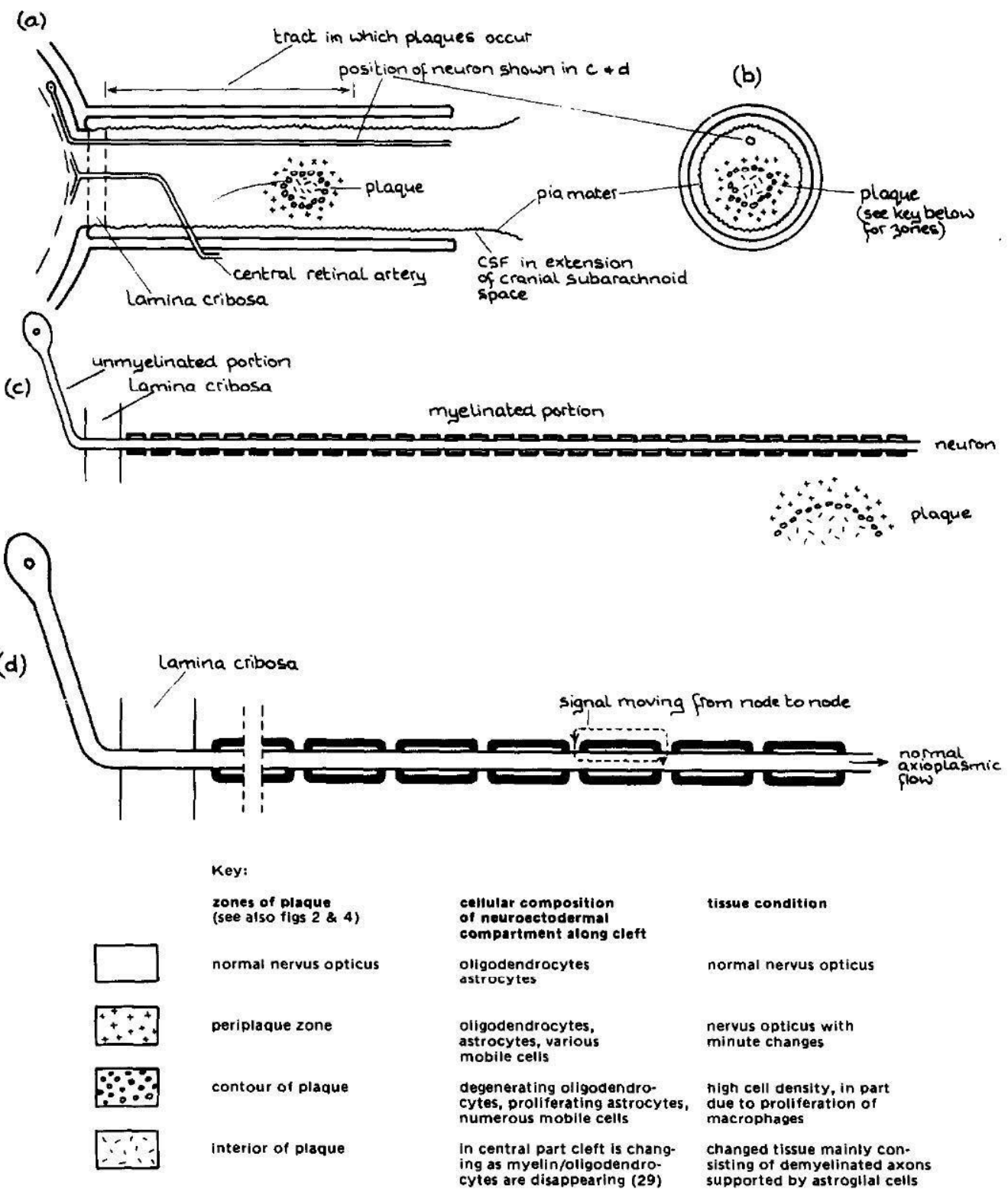

Fig 3 Presumed subclinical MS-ON pathology

(a) Longitudinal section of optic canal showing optic nerve ensheathed in pia. A small plaque is indicated as having developed at some distance from the lamina cribrosa.

(b) Cross section of optic canal showing optic nerve with a small central plaque.

(c) Diagramatic representation of neuron indicated in (a) and (b) showing unmyelinated and myelinated portion.

(d) Diagramatic representation of neuron indicated in (a), (b) and (c) showing principle of signal transmission (32). In this diagram and in the diagrams of fig. 4, (b), (c) and (d) each myelin sheath shown stands in for several as diameters are large out of proportion.

The rough outline given above constitutes a variant theory concerning CNS-reaction to methanol. This is worth discussing as it could be tested. The technology exists to evaluate longterm toxicity of sub-lethal doses (12, p 18 \& 45).
MS, which occurs in populations in which common food habits induce 'methanol in blood' episodes rather often, has been postulated to be due to the selfsame toxic-allergic mechanism (11, $12,13,29)$. In the following we will try to test 

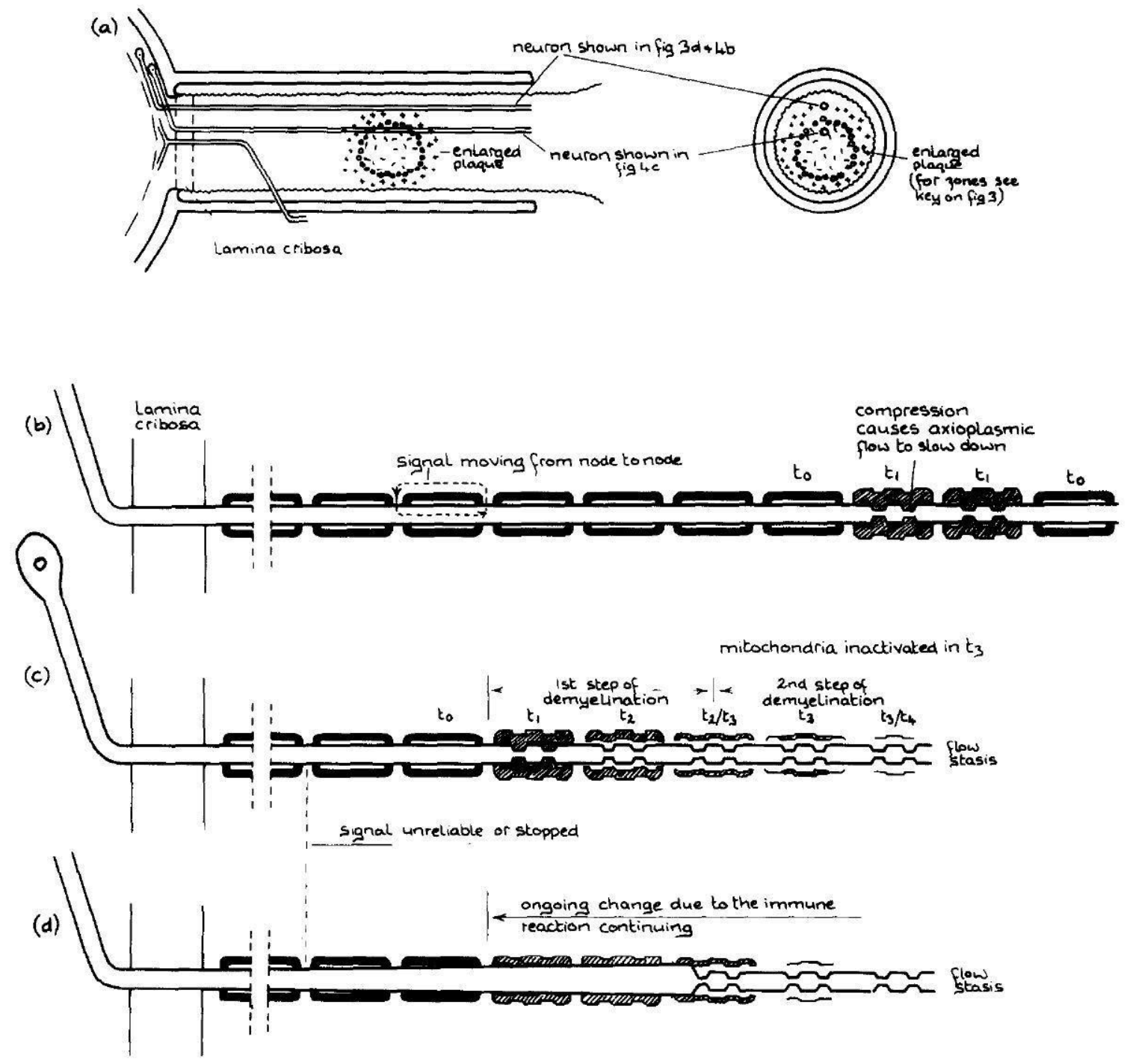

Fig 4 Presumed pathology when symptoms of $\mathrm{ON}$ are impending

(a) shows the plaque as per fig. 3 grown larger so that symptoms of neuritis retrobulbaris are impending

(b) shows the same neuron as fig $3 \mathrm{~d}$ with swollen myelin sheaths where passing through the periplaque zone (see fig. 2 \& 3)

(c) shows the changes in myelin sheaths and axon of a neuron as it enters the plaque from left to right (see fig. 2 for symbols of myelin sheaths)

(d) shows the same neuron as (c) later in time with axoplasm mmed up due to continued flow stasis

whether the MeHyp-model can elucidate some aspects of the coeco-central optic neuritis symptom in MS.

Demyelination in the retrobulbar optic nerve during the premorbid period

The local expression of the toxic-allergic process in the retrobulbar optic nerve is considered in Figure 3 for a sensitized individual. At present this person has no clinical evidence of disease, but has the food habit and other factors that bring about repetitive 'methanol in blood' episodes. In Figure 3 it is assumed that a minute plaque has formed. The neuron chosen for diagramatic representation in Figure 3d bypasses this plaque with sufficient margin to be unaffected. During later 'methanol in blood' episodes the plaque will increase in size. Such a plaque 
(a)

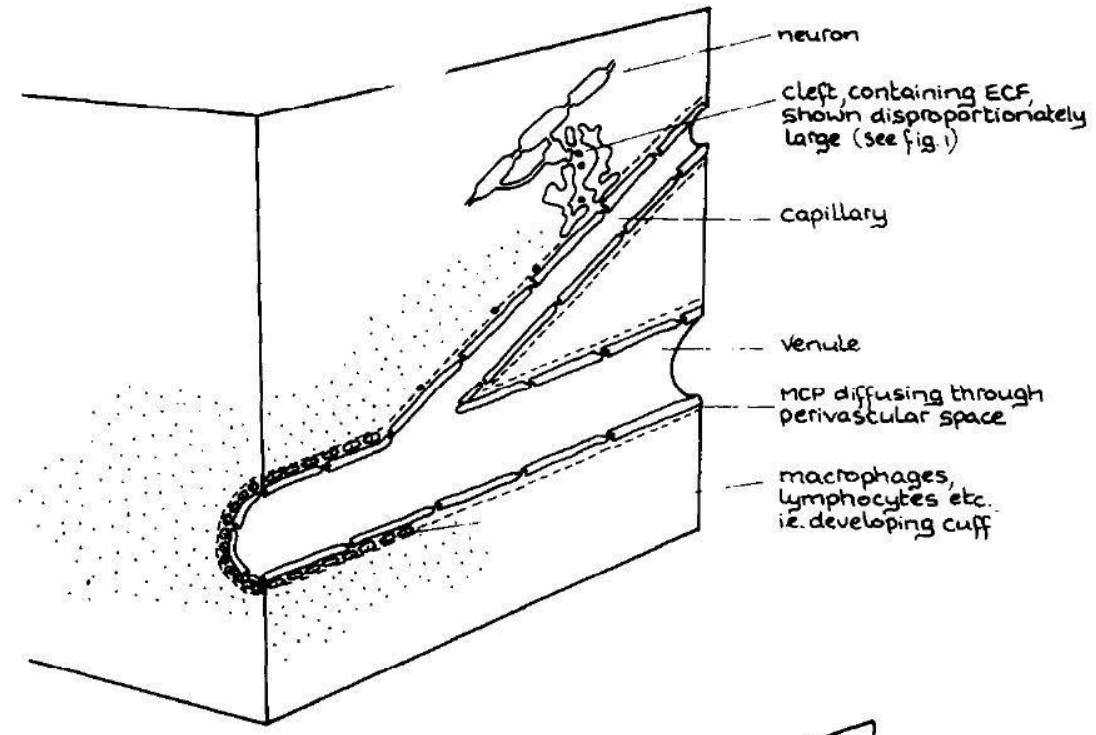

(b)

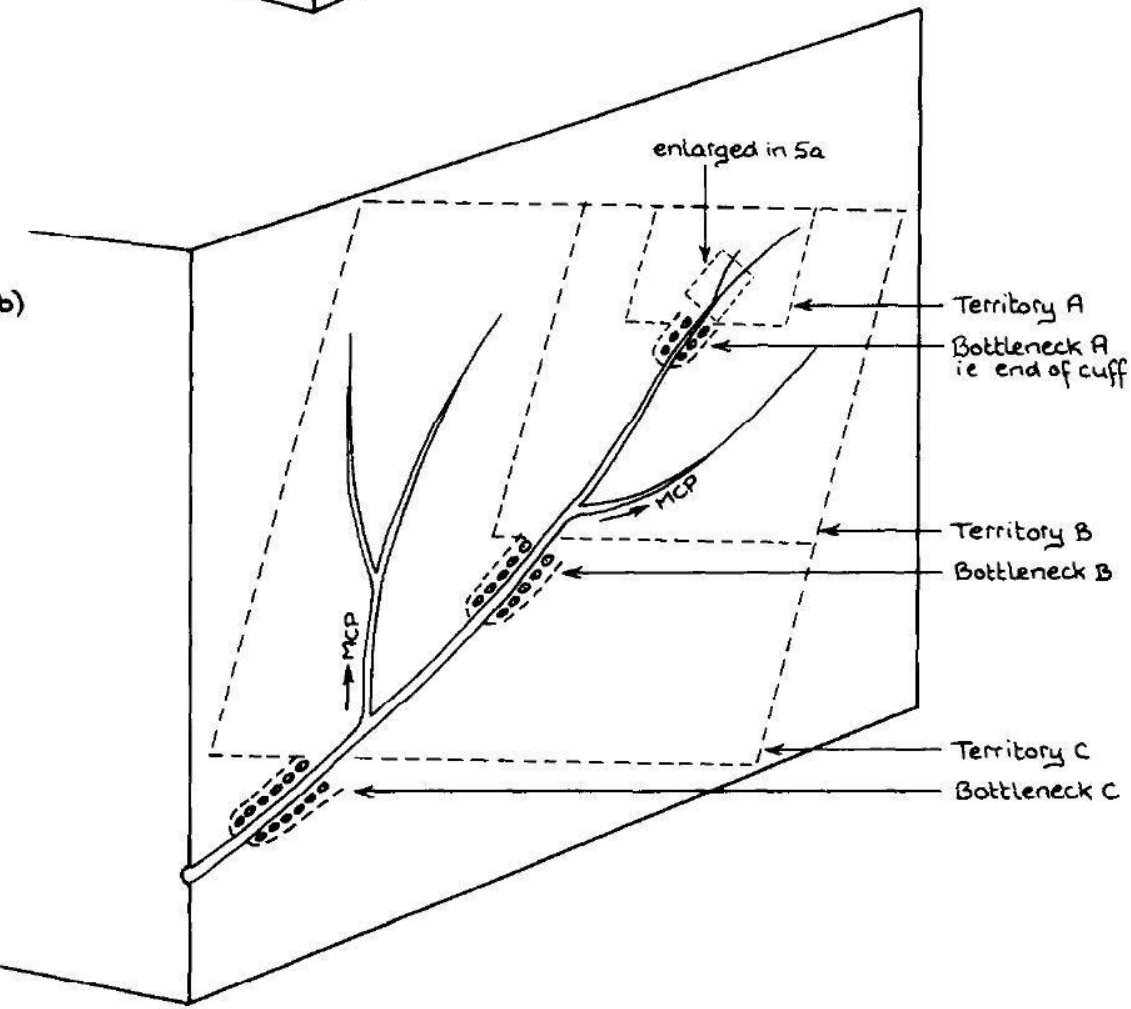

Fig 5 Visualisation of mechanism of damming back MCP (pathologic ECF) into venous drainage territories

(a) Perspective view of the setting of fig. 1 along a capillary/venule indicating accumulation of mononuclear cells.

(b) Showing the principle of bottlenecks in perivascular space (accumulations of mononuclear cells extending downstream) involving ever larger venous drainage territories.

is drawn in Figure $4 \mathrm{a}$ and due to the expansion the neuron now passes through the periplaque zone. In the diagramatic representation (as Figure 4b) some myelin sheaths have become swollen and are indicated by the symbol corresponding to stage $t_{1}$. Stage $t_{1}$ occurs, as can be seen from Figure 2, when MCP at a node of Ranvier rises above the homeostatic limit. 
Simultaneously methanol/formaldehyde will transform, as explained above under $\mathrm{i}$ to iv, myelin protein or glycoprotein into antigen and thus initiate in the sheaths affected the first step of demyelination (see Fig. 2, top diagram to the right of centre). The swelling will cause some compression of the axon and a slow down of axoplasmic flow (10). Axolemma and membranes of mitochondria would be under the influence of MCP as well, but in the same way as other membranes in the body at that time would maintain their function under the adverse condition. Thus signalling would for the time being continue normally.

In Figure $4 \mathrm{c}$ a neuron passing through the contour of the plaque is shown, in which the various symbols indicative of pathologic change corresponding to stage $t_{0}$ to $t_{3} / t_{4}$ transition of Figure 2 occur. The neuron passes through a number of clefts and meets in each a more intense degree of changes. As the immune reaction becomes more intense (compare MCP level at $t_{1}$ with $t_{2} / t_{3}$ transition in Fig. 2) the harmful effects of compression (17) and pathologic ECF (eodema) bring about myelinolysis, stasis of axoplasmic flow and unreliable or interrupted signalling. The information on sodium pump activity on the far right on Fig. 2 is taken from research findings (14) on enzymatic activity in MS plaques.

It is to be noted that the diagram at the top right of centre in Figure 2 has a question mark in stage $t_{4}$. This expresses the difficulty of judging conditions in the interior of the plaque. Oligodendrocytes are disappearing in this area and astroglial processes proliferate. The denuded axons are mostly able to maintain themselves and there is an indication that eventually enzymatic activity in the axons will recover. Inside the plaque (in the denuded axons) the enzymatic activity is higher than in normal white matter (14).

It is important to note that Figure 2 and the diagramatically shown neurons in Figure $4 b$ and $4 \mathrm{c}$ reflect conditions at the height of a 'methanol in blood' episode. Such an episode passes in a few hours and the pathologic ECF will, where methanol was mainly responsible for the deviation, return to homeostasis. However in clefts (Figures $1 \& 5$ ) where MCP is influenced by the immune reaction, minute change will continue. Conduction may be unreliable in neurons where: i) $\mathrm{MCP}$ causes an unsuitable microclimate at nodes of Ranvier; ii) myelin sheath has been completely removed during the recnt 'methanol in blood' episode;

iii) compression combined with high MCP has induced extended flow stasis in axolemma (swollen axon see Fig. 4d).

However apart from these the vast majority of neurons in the optic nerve continue to signal normally. This means that a plaque as shown in Figures $3 a$ or $4 a$ does not cause a clinical symptom.

Acute optic (retrobulbar) neuritis as a result of pathologically changed extra cellular fluid involving a large part of the cross section of the nerve

As discussed above, a plaque as shown in Figures $3 \mathrm{a}$ and $3 \mathrm{~b}$ would, after the next "methanol in blood' episode, enlarge due to the complexities of the immune reaction. Extension of cuffs out of the lesion along the course of venules, i.e. Dawson's finger, is a feature of the contour (1). Gradually more neurons become involved and enter a period of unknown duration of unreliable or interrupted signal conduction.

The number of neurons affected being relatively low, the patient can be expected to be and remain asymptomatic whilst the plaque grows in the next few months or years to a size as shown in Figures 4a. Even in this sizeable plaque, with a large periplaque zone, the axons affected by the stage $t_{3}$ mechanism would not be more than about $30 \%$ of the total. Obviously with $70 \%$ of neurons signalling normally the symptom of blindness can not arise.

Moreover the ' $O N$ ' bout with a forerunner symptom of blurred vision on taking exercise and the severe symptom coming on over night or (in other cases) spread out over several days or longer has seemingly a different and more varied time course than growth at the contour. This may involve additional clefts changing from first step of demyelination to myelinolysis (stage $t_{3}$ ) in the second step. Figure $5 \mathrm{~b}$ brings in an aspect which has hitherto not been emphasized. Whenever demyclination is active in some of the clefts of a venous drainage territory, lymphocytes and macrophages etc. may produce a bottleneck at more or less any point along the vessel, which can have dramatic consequences.

The concept discussed in Figure 1c requires - in order to maintain homeostasis at the nodes of Ranvier - that molecules can readily diffuse into CSF, which in the optic nerve means diffusion through the collagen and astroglia tissue in 
the peri-vascular space along a tortuous route into the CSF which surrounds the pia mater (2). If, due to demyelination, a bottleneck situaltion develops (see Figure 5) in a more distal part of the perivascular space of the vein this is a serious matter.

Under these circumstances pathologic ECF (containing MCP composed of methanol and formate and lysosomal enzymes etc.) will begin to diffuse and induce a sequence of events starting with penetration of MCP into large numbers of hitherto unaffected clefts (see Fig. $1 \& 5$ ) in the vein's drainage territory. Astrocytes will increase molecular pumping but outflow will be restricted until somewhere along the perivascular space an emergency outlet develops. Thus some part or a large section of the glial and connective tissue around the blood vessels becomes distended.

Thus, we believe, the acute phase of $\mathrm{ON}$ is induced by oedema, membrane attacks and selfagravating myelinolysis in the drainage territory of the vein or veins affected by the critical accumulation of the cells of the immune reaction. Symptoms of ON appear as the oedema spreads into larger areas either swiftly over night or more slowly over several days or weeks. Venous sheathing as observed in the fundi of about $10 \%$ of MS patients $(15,31)$ is possibly an aftereffect of such diffusion. The low figure of $10 \%$ can be understood; the normal truly retrolaminar position of the plaque involves veins not open to funduscopic observation.

The symptom of optic neuritis in relation to the subclinical and clinical course of the disease

Figure 6 in an interpretation of development of $\mathrm{ON}$. The acute symptom is experienced during the last 3 days, when between 80 and $95 \%$ of neurons have become unreliable signal transmitters. Only during these 3 days was the patient (9) aware of the disease condition, which, if one considers its mechanism, must have had it's beginning a long time previously. The graph is speculative but usefully illustrates the disease process under discussion.

The dotted line in the upper graph denotes the percentage of neurons which have become unable to signal normally, because they pass through the contour of the plaque (axonal mitochondria inactivated in stage $t_{3}$, see Fig. $2 \& 4$ ). The solid line indicates additional neurons which are unable to signal normally. The ordinate between the dotted and the solid line therefore indicates the percentage of neurons in which functional blocking occured due to secondary oedema. In these neurons defective signal transmission is considered to have been caused by MCP from a zone in stage $t_{3}$ diffusing into adjacent clefts (see Fig. 1, arrow at left indicates normal movement through perivascular space into CSF, the diffusion during secondary oedema is movement in the opposite direction) of the venous drainage territory (see Fig. 5).

The lower graph of Figure 6 shows the time course subdivided into a number of periods between the appearance of the first minute changes and acute $\mathrm{ON}$. This shows how in the plaque forming period all three stages $t_{1}, t_{2}, t_{3}$ occur simultaneously and that the diffusion of pathologic ECF brings about the acute phase. It is important to note that the premorbid period is very long as compared to this latter event. 9 years have been chosen in the graph because studies in immigrants (discussed in $(12$, p 32 \& 37) have shown that MS develops normally about 9 to 13 years after primary sensitization. However development can be much faster. An MS case of a child is on record (8) in which gait inbalance was a symptom at 2 years 11 months of age.

Such observations suggest that habits and personal metabolism determining frequency, intensity and time course of 'methanol in blood' episode play a role. Another important variable is the individual immunological reaction. Current research into oligoclonal banding in monosymptomatic MS patients may turn out to give some knowledge of an individual's immune reaction status (21).

Our model can now be compared with hitherto held views on ON: Hayreh (10) gave the explanation: 'In retrobulbar optic neuritis the demyelinating lesion may be localized, but the association oedema may be more widespread to involve most of the nerve transversely, resulting in a transverse compression of the axons because of the nonelastic fibrous nature of the optic nerve architecture'.

In this sentence the essential mechanism has been set out in an admirably clear manner. In the MeHyp-model some auxiliary phenomena are under review: i) growth of plaque,

ii) how the associated oedema forms and becomes widespread,

iii) the mechanisms of volume-increase which lead to the transverse compression: 
(a)

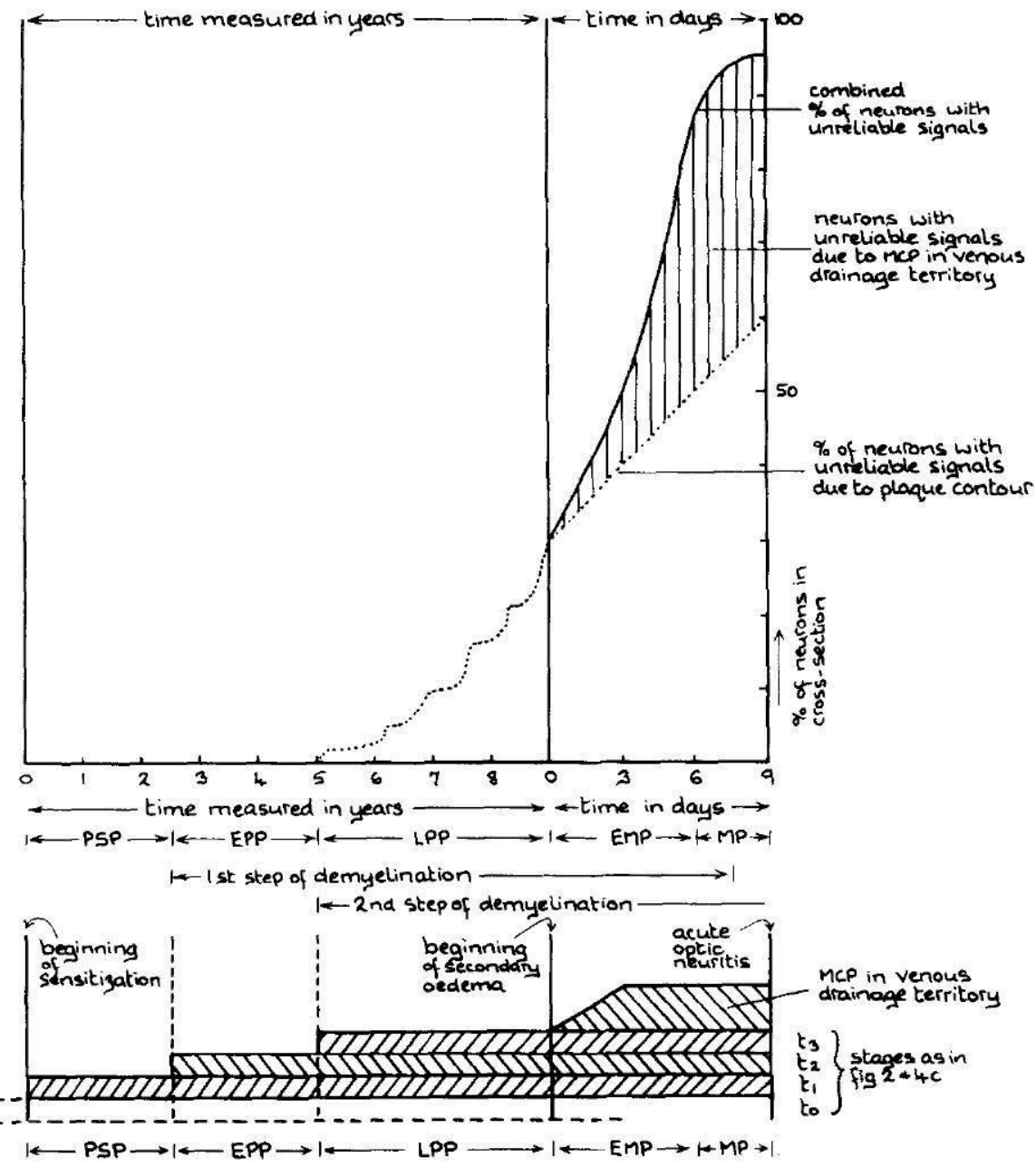

Fig 6 Events leading to the symptom on ' $O N$ '

(a) Relationship between the course of the disease and \% neurons in retrolaminar optic nerve experiencing 'unreliable signal conduction:.

Key to epochs:

PSP - Primary sensitizing period, 'Methanol in blood episode' causing ECF-microclimate as per stages $t_{0}$ and $t_{1}$ (see fig. 2)

EPP - Early permorbid period, ECF microclimate as per $t_{0}, t_{1}$ and $t_{2}$. Immune reaction active; lymphocytes and macrophages etc. appear in perivascular space in focal points along venules. Patient by medical criteria in perfect health, although 1st step demyelination occurs. At this stage the patient is fully sensitized for MS.

LPP - Late premorbid period, ECF microclimate as per $t_{1}, t_{2}, t_{3}$. Immune reaction very active; due to accumulation of macrophages etc., bottleneck situations occur along perivascular space of venules. Patient considered to be in perfect health, although 1st step and 2 nd step demyelination occurs and a small plaque develops in the retrolaminar nerve.

EMP - Early morbid period, ECF microclimate as per stage $t_{3}$ in plaque contour and as per stages $t_{1}$ and $t_{2}$ in peri plaque zone. $\mathrm{A}$ bottleneck has developed in the perivascular space of a vein; therefore $\mathrm{MCP}$ as occuring at plaque contour begins to influence nodes of Ranvier in the venous drainage territory. Patient experiences fatigue and may have speels of blurred vision.

MP - Morbid Period, ECP etc. as in EMP; the cross section around the plaque comes increasingly under the influence of MCP. Patient experiences acute 'ON'. In the case quoted (9) blurred vision occured in the left eye three days before visual acuity went down to counting fingers.

(b) Diagram showing type of ECF abnormality during epochs. 4 types of abnormality are distinguished. In addition stage Note:

$t_{0}$ is shown. At this stage methanol diffuses into perivascular space but homeostasis at node of Ranvier is not yet affected.

This is an attempt at interpretation. The increasing number of neurons with unreliable conduction properties during the last three days (morbid period) is taken from a well studied case (9), but the information presented for the earlier epochs is surmised in line with general knowledge of the MS syndrome (see text). 
(a) swelling of myelin due to diffusion of MCP (see Fig. 2),

(b) swelling of axons as an after-effect of a) (see Fig. 4d). Is it possible that we are reaching the basis of a deterministic explanation of acute $\mathrm{ON}$ ?

\section{Summary and perspectives}

Research findings have allowed us to consider the effects of abnormal stimuli exerted on brain tissue by endogenously occuring substances. As discussed before (29) the diffusion of methanol through the walls of brain blood vessels will occur, as this low molecular weight substance distributes in the tissues pro rata with their water content. This may, depending on auxiliary factors and the patient's metabolism, if blood methanol level is episodically above the homeostatic range, lead to a complex cascade of events including an immune reaction.

The concept has been evolved around a case history (13) which sounds like an involuntary experiment on man. ON-MS was induced by repetitive inhalatory intoxication with quantities of absorbed methanol on each occasion approximately in the range of 10 to $30 \mathrm{mg} / \mathrm{kg}$ bodyweight.

Such modest quantities of toxin (which in some persons may occur due to metabolic processes) can - via the interplay of toxic-alleric processes - cause subtle changes. One of these is that extra-cellular fluid (ECF) near nodes of Ranvier of retrolaminar optic nerve becomes abnormal. Methanol, methanol's metabolites and various mobile cells and cellular degradation products start to occur in ECF. These abnormal ingredients have been termed 'molecules characterizing pathology' (MCP). MCP varies in concentration and composition during the course of the disease.

In microscopic situations along venules, MCP concentration is at times drastically changed because a vital drainage channel has bee plugged; consequence are myelinolysis and inactivation of axonal mitochondria of neurons in the neighbourhood. Signal conduction in the neurons affected becomes unreliable as the energy requirements of the potassium/sodium pump can no longer be met. This happens, when immunological activity has been high, as an aftereffect of a 'methanol in blood' episode.

Many episodes play out this scenario silently but gradually a small plaque arises which grows at the contour. Ultimately the critical 'methanol in blood' episode occurs which translocates the plug from the perivascular space of a small vessel to that of a vein. The pattern of observations in acute $\mathrm{ON}$ and in histopathological studies permits one to conclude that at this stage a rapid increase of oedema occurs, often affecting the entire cross-section near the plaque. Thus very large numbers of myelinated fibres experience compression, axoplasmic flow stasis, unreliable signal transmission and so the silent premorbid process becomes within hours or days acute optic neuritis.

During the time of the acute attack the body's cells degrade/eliminate the toxin and its metabolites and repair processes get under way.

After the symptoms of ON have been at their worst, extracellular fluid may normalize. Thus the neurons outside the plaque contour will under favourable circumstances again signal normally. In those passing through the contour some internodes will have become demyelinated during the recent 'methanol in blood' episode; others will have been partly demyelinated.

There are a number of elegant techiniques which can be used to monitor neuron behaviour in vivo during the weeks following on acute attack $(6,7,20,23,31,33)$. The patterns observed make it probable that several mechanisms are involved in the very variable recovery from acute $\mathrm{ON}$, termed remission. Research by a number of authorities $(14,27,32)$ gives guidelines as to how some aspects might be clarified.

Brain physiologists are discussing the role of membranes, and cytoplasm of endothelial cells of blood vessels $(16,24,35)$ in the homeostatic control of various ions and small molecules diffusing into ECF (3). The open arrows in Fig. 1 indicate what would well be the access route used in homeostasis by the self-same molecules which in hypernormal quantity can induce pathology.

It is submitted that the methanol hypothesis model elucidates various hitherto unexplained phenomena and gives a set of parameters to those interested in the nature of perivenous sheathing $(23,31)$ and of immunopathology associated with selective host cell destruction (34).

Such studies may reinforce the clues presented. It appears probable that analysis of metabolic interrelations and homeostasis will lead towards understanding of optic neuritis as occuring in multiple sclerosis. 


\section{Reference}

1. Adams C. W. M. Pathology of Multiple Sclerosis. Progression of the Lesion. Brit. Med. Bull. 33: 15-20. 1977.

2. Anderson D. R. \& Hoyt W. F. Ultrastructure of intraorbital portion of human and monkey optic nerve. Arch Ophthal 82: 506-530, 1969.

3. Brown W. Jann. The capillaries in acute and subacute MS plaques, a morphometric analysis. Neurology 28(2): 84-92, 1978.

4. Ferry D. G., Temple W. A., McQueen E. G. Methanol Monitoring. Int Arch Occup Environ Health 47: 155-165, 1980 .

5. Friede R. 1. Enzyme histochemistry of neuroglia. in 'porgress in brain research' 15 (E.D. De Robertis \& R. Carrera ed.) Elsevier, 1965.

6. Galvin R. J., Regan D., Heron J. R. A possible means of monitoring the progress of demyelination in multiple sclerosis: effect of body temperature on visual perception of double light flashes. J. Neuroll. Neurosurg. Psychiatry 39(9): 861-865, 1976.

7. Green J. B., Wolcoft M. R. Evoked Potentials in multiple sclerosis 1982 Arch Neurol 39: 696-697.

8. Hauser S. I.. Bresnan M. J., Reinherz E. L., Weiner H. L. Childhood multiple sclerosis: Clinical features and demonstration of changes. Annals of Neurology 11: 46.3-468, 1982 .

9. Halliday A. M. \& McDonald W. I. Pathophysiology of Demyelinating Disease, Brit. Med. Bull. 33: 21-26, 1977.

10. Hayrch S. S. Optic disc edema in raised intracranial pressure; pathogenesis, Arch Ophthal 95: 1553-1565. 1977.

11. Henzi Hugo. Methanol und Nebenfaktoren als Ursache einer praemorbiden Phase der multiplen Sklerose mit Retrobulbaärneuritis. Juris Druck, Zürich, 1968.

12. Henzi Hugo. The Methanol Hypothesis. A new concept of multiple scierosis: The path to causal treatment, report on 80 cases. Juris Druck. Zürich. 1980.

13. Henzi Hugo. Chronic Methanol Poisoning with the clinical and pathologic-anatomical features of multiple sclerosis. Medical Hypotheses 13: 63-75, 1984.

14. Hirsch Hilde E. \& Parks Mary E. $\mathrm{Na}^{+}$-and $\mathrm{K}^{+}$dependent Adenosine Triphosphatase changes in multiple sclerosis plaques. Annals of Neurology 13: 658-663. 1983.

15. Hollwich Fritz. Augenheilkunde, 9th edition George Thieme Stuttgart

16. Kuffler Stephen W. \& Nicholls John G. From Neuron to Brain; a cellular approach to the function of the nervous system. Sinnauer Associates Inc Publishers Sunderland Mass, 1976

17. Levy N. S. Axoplasmic Transport in the visual system. In current concepts of ophtalmology, (H. E. Kaufman \& T. J Zimmerman ed.) Mosby Co Saint Louis, 1976.
18. Lumsden C. E. In: Multiple Sclerosis a reappraisal 2 nd ed. by McAlpine D., Lumsden C. E.. Acheson E. D., Churchill Livingstone, Edinburgh, 1972.

19. Majchrowicz E. Biochemical Pharmacology of ethanol p 122 Plenum Press New York, 1975.

20. McDonald W. I. Acute Optic Neuritis, Brit I Hospital Medicine 42-48. July 1977.

21. Moulin Dwight. Donald W. Paty and Ebers George C. The predictive value of cerebrospinal fluid electrophoresis in 'possible' multiple sclerosis, Brain 106: 809-816. 1983.

22. Nicholls P. Formate as an inhibitor of cytochrome oxidase, Biochemistry. Biophysic Res Commun 67: 610-616, 1975.

23. Nikoskelainen Eeva, Folek Björn. Do visual evoked potentials give relevant information to the neuro-ophtalmological examination in optic nerve lesions? Acta neurol Scandinav 66: 42-57, 1982.

24. Oldendorf William $H$. The Blood Brain Barrier Exp. Eye Res. Supp. 177-190, 1977.

25. Perrier $O$. Lesions anatomo-pathologiques des voies optiques et du tronc cerebral dans la sclerose en plaque. Bull Socbelge Ophtal, 199-200: 83-94, 1982.

26. Prineas J. W. \& Wright R. G. Macrophages, Lymphocytes and Plasma Cells in the perivascular compartment in chronic multiple sclerosis. Laboratory investigation 38: 409-421. 1978.

27. Rasminsky $M \&$ Sears T. A. Internodal conduction in undisected demyelinated nerve fibres. J. Physiol 227: 323-350. 1972.

28. Sargent D. Personal communication, 1982

29. Schwyzer R. W. \& Henzi Hugo. Multiple Sclerosis: Plaques caused by 2-step demyelination? Medical Hypotheses 12: 129-142, 1983.

30. Sedivec V., Mraz M., Flek J. Biological monitoring of persons exposed to methanol vapours. Int Arch Occup Environ Health 48: 257-271, 1981.

31. Toussaint D. Altération du fond doeil dans la sclerose. en plaques Bull Soc belge Ophtal, 199-200), 235-256. 1982.

32. Waxman Stephen G. Current concepts in Neurology, Membranes. Myelin and the Pathophysiology of Multiple Sclerosis, The New England Journal of Medicine 306: 1529-1533, 1982.

33. Young I. R., Pallis C. A., Bydder G. M., Hall A. S., Legg N. J., Steiner R. E. Nuclear Magnetic Resonance Imaging of the brain in multiple sclerosis. The Lancet 14 Nov 1981.

34. Zinkernagel R. M. Role of virus-induced cell-mediated immunereactions, Verh. Dtsch. Ges. Path. 65: 11-14. 1981.

35. Zinn K. M. \& Leopold 1. H. Molecular Biology of the cell in ocular fine structure for the clinician, edited by K. M. Zinn, Little, Brown \& Co, Boston, 1973 